\title{
Brand logo design: examining consumer response to naturalness
}

\author{
School of Economics and Management and CEGE, Universidade Católica Portuguesa, Porto, \\ Portugal \\ Leonor Vacas de Carvalho \\ Departamento de Gestão, CEFAGE-UE, Escola de Ciências Sociais, Universidade de Évora, \\ Évora, Portugal \\ Anna Torres \\ Department of Economics and Business, Universitat Pompeu Fabra, Barcelona, Spain, and \\ Patrício Costa \\ School of Health Science, Minho University, Braga, Portugal
}

\author{
Abstract \\ Purpose - This paper aims to study how logo design characteristics influence consumer response. Based \\ on an in-depth literature review on \\ consumer responses to logo design, the authors included in this research one fundamental dimension of \\ logo design, namely, naturalness and \\ investigated the influence of the different types of natural logo designs on affective response. \\ Design/methodology/approach - In total, 96 logos were selected as design stimuli. The logos were \\ previously classified, according to the \\ naturalness of the logo design, as having an abstract, cultural or organic design. Responses were \\ gathered through a survey in Portugal, including \\ two studies with 220 participants. \\ Findings - Results show that naturalness is an essential logo design element which significantly \\ influences consumer affective responses to the \\ logo, and that natural logos are clearly preferred to abstract logos. Additionally, this research indicates \\ that, within natural logos, organic designs \\ are favored over cultural designs. \\ Practical implications - The findings presented suggest that affect toward unknown organic logos is at \\ the same level as affect toward \\ well-known abstract logos. This is a relevant finding from a managerial point of view, as familiarity, an \\ essential cognitive response toward the brand \\ that has a cost for the firm, can be replaced cost-free with unknown organic logos. \\ Originality/value - This paper is a first exploration of responses to different types of natural logo design. \\ The results should guide managers in \\ selecting or modifying logo designs for achieving a positive affective response. \\ Keywords Logo design, Brand logos, Consumer response
}

